\title{
Entanglement Entropy in a Holographic Moving Mirror and the Page Curve
}

\author{
Ibrahim Akalø ${ }^{1}$ Yuya Kusuki $\odot,{ }^{1}$ Noburo Shiba, ${ }^{1}$ Tadashi Takayanagi $\odot,{ }^{1,2,3}$ and Zixia Wei ${ }^{1}$ \\ ${ }^{1}$ Yukawa Institute for Theoretical Physics, Kyoto University, Kitashirakawa Oiwakecho, Sakyo-ku, Kyoto 606-8502, Japan \\ ${ }^{2}$ Inamori Research Institute for Science, 620 Suiginya-cho, Shimogyo-ku, Kyoto 600-8411, Japan \\ ${ }^{3}$ Kavli Institute for the Physics and Mathematics of the Universe, University of Tokyo, Kashiwa, Chiba 277-8582, Japan
}

(Received 8 December 2020; accepted 25 January 2021; published 12 February 2021)

\begin{abstract}
We calculate the time evolution of entanglement entropy in two-dimensional conformal field theory with a moving mirror. For a setup modeling Hawking radiation, we obtain a linear growth of entanglement entropy and show that this can be interpreted as the production of entangled pairs. For the setup, which mimics black hole formation and evaporation, we find that the evolution follows the ideal Page curve. We perform these computations by constructing the gravity dual of the moving mirror model via holography. We also argue that our holographic setup provides a concrete model to derive the Page curve for black hole radiation in the strong coupling regime of gravity.
\end{abstract}

DOI: 10.1103/PhysRevLett.126.061604

Introduction.-Moving mirrors have been known for a while as a class of instructive models that mimic Hawking radiation [1] based on quantum field theory [2,3] where unitarity is manifest. On the other hand, in the case of black hole evaporation, it has been a significant problem to understand whether unitarity is maintained in the gravitational theory. One manifestation of unitary black hole evaporation is the Page curve for the entropy of Hawking radiation [4]. Based on the fine grained entropy formula [5-8], this has been derived semiclassically for field theories coupled to gravity [9-11] and confirmed by direct gravity replica computations [12,13]. See, e.g., Refs. [14-62] for further progress along this direction. For recent related works refer to Refs. [63-65].

In this Letter, we first present concrete calculations of entanglement entropy in moving mirror setups and show that this leads to an ideal Page curve. Moreover, we present a close connection between moving mirror models and black hole radiation via a particular version of the anti deSitter (AdS)/conformal field theory (CFT) correspondence [66], namely, in the case when the CFT is defined on a manifold with a boundary $[67,68]$. For earlier studies of entanglement entropy in moving mirror models refer to Refs. [69-73].

At the same time, our moving mirror models provide a class of new nonequilibrium setups, where quantum entanglement evolves rapidly. Quantum quenches [74] have attracted much attention in broad communities including

Published by the American Physical Society under the terms of the Creative Commons Attribution 4.0 International license. Further distribution of this work must maintain attribution to the author(s) and the published article's title, journal citation, and DOI. Funded by SCOAP ${ }^{3}$. condensed matter, quantum information, and high energy. Our setups provide a new version of quantum quenches, which have very rich structures of quantum entanglement and which can even be realizable experimentally. In addition, interestingly, the formulation of a moving mirror is interpreted as a novel generalization of boundary conformal field theory (BCFT) as we will point out later.

A moving mirror from conformal maps.-A moving mirror setup in two dimensions is specified by the trajectory of a mirror profile $x=Z(t)$. We consider a CFT which lives on the right region, i.e., $x \geq Z(t)$. A conformal transformation (here, we set $u=t-x$ and $v=t+x$ ) [2,3],

$$
\tilde{u}=p(u), \quad \tilde{v}=v
$$

maps this into a simple setup with a static mirror $\tilde{u}-\tilde{v}=0$, as depicted in Fig. 1. Here, we choose the function $p(u)$ such that the mirror trajectory is given by $v=p(u)$, i.e.,

$$
t+Z(t)=p[t-Z(t)]
$$
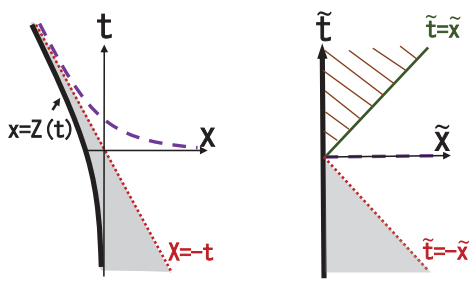

FIG. 1. The moving mirror setup (left) and its conformal transformation into a static mirror (right). The mirror trajectory is depicted by the thick curve. The shaded region shown in the right panel corresponds to an inside horizon region, which is missing in the left picture. 
For example, we can calculate the energy stress tensor from the conformal anomaly via the map Eq. (1), such that

$$
T_{u u}=\frac{c}{24 \pi}\left[\frac{3}{2}\left(\frac{p^{\prime \prime}(u)}{p^{\prime}(u)}\right)^{2}-\frac{p^{\prime \prime \prime}(u)}{p^{\prime}(u)}\right],
$$

where the components $T_{u v}$ and $T_{v v}$ are vanishing.

As an example of a CFT, consider a massless free scalar $\phi$. We impose the Dirichlet boundary condition $\phi(t, Z(t))=0$ along the mirror trajectory. A complete set of positive frequency solutions to the equations of motion $\partial_{u} \partial_{v} \phi=0$, which satisfy the latter boundary condition, reads

$$
\phi_{\omega}(t, x)=i(4 \pi \omega)^{-1 / 2}\left(e^{-i \omega v}-e^{-i \omega p(u)}\right) .
$$

Then, $\phi$ can be expanded in terms of these modes [2] as

$$
\phi(t, x)=\int_{0}^{\infty} d \omega\left[a_{\omega}^{\mathrm{in}} \phi_{\omega}+a_{\omega}^{\mathrm{in} \dagger} \phi_{\omega}^{*}\right]
$$

where $a_{\omega}^{\text {in }}$ and $a_{\omega}^{\text {in } \dagger}$ are the annihilation and creation operators, respectively. The in-coming vacuum $\left|0_{\text {in }}\right\rangle$ is defined by the state annihilated by $a_{\omega}^{\text {in }}$ and the out-going vacuum $\left|0_{\text {out }}\right\rangle$ is given by a Bogoliubov transformation of $\left|0_{\text {in }}\right\rangle$. The expectation value of the energy stress tensor, i.e., $\left\langle 0_{\text {in }}\left|T_{u u}\right| 0_{\text {in }}\right\rangle$, reproduces Eq. (3) for $c=1$.

A moving mirror with horizon.-For a typical example which models Hawking radiation from a black hole, we choose

$$
p(u)=-\beta \log \left(1+e^{-\frac{u}{\beta}}\right),
$$

where the parameter $\beta$ plays the role of an inverse temperature. Its profile is depicted in the left picture of Fig. 1. In the early time limit $t \rightarrow-\infty$, we have $Z(t) \simeq 0$, while in the late time limit, the mirror trajectory gets almost lightlike, $Z(t) \simeq-t-\beta e^{-2 t / \beta}$. As depicted in Fig. 1, the region $\tilde{u} \geq 0$ in the extended coordinates is missing for the original coordinates. This region is analogous to the inside horizon region in the black hole formation process.

The energy stress tensor [Eq. (3)] reads

$$
T_{u u}=\frac{c}{48 \pi \beta^{2}}\left(1-\frac{1}{\left(1+e^{u / \beta}\right)^{2}}\right),
$$

which vanishes at early time $u \rightarrow-\infty$, and becomes a constant thermal flux, $T_{u u} \simeq c / 48 \pi \beta^{2}$, at late time $u \rightarrow \infty$.

Let us calculate the entanglement entropy $S_{A}$ for a semiinfinite subsystem $A$ given by $\left[x_{0}, \infty\right]$ at time $t$. We can calculate $S_{A}$ from the one point function of the twist operator [75-77] on the upper half plane $\tilde{x}>0$ by using the conformal map [Eq. (1)] via the replica method. We find

$$
S_{A}=\frac{c}{6} \log \frac{t+x_{0}-p\left(t-x_{0}\right)}{\epsilon \sqrt{p^{\prime}\left(t-x_{0}\right)}}+S_{\mathrm{bdy}} .
$$

Here, $\epsilon$ is the UV cutoff (lattice spacing) of the CFT and $S_{\text {bdy }}$ is the boundary entropy $[68,75,78]$. Note that this formula holds for any CFT.

If we fix the end point of the subsystem $A$, i.e., $x_{0}$, we can approximate Eq. (8) at late time $t \rightarrow \infty$, and find

$$
S_{A} \simeq \frac{c}{12 \beta}\left(t-x_{0}\right)+\frac{c}{6} \log \frac{t}{\epsilon}+S_{\text {bdy }} .
$$

The first term linear in $t$ arises from entangled pair production due to the moving mirror, while the second $\log t$ term comes from the standard vacuum entanglement as the length of the complement of $A$ grows linearly.

To study the first contribution in more detail, we allow changing the value of $x_{0}$ time dependently as

$$
x_{0}(t)=-t+\xi_{0} .
$$

In the late time limit $u \rightarrow \infty$, we obtain

$S_{A}=\frac{c}{6} \log \frac{\left(\xi_{0}+\beta e^{-\left(t-x_{0}(t)\right) / \beta}\right) \sqrt{1+e^{\left(t-x_{0}(t)\right) / \beta}}}{\epsilon}+S_{\mathrm{bdy}}$.

We choose $\xi_{0}$ to be positive, but sufficiently small. If the left end point of $A$, given by $(u, v)=\left(2 t-\xi_{0}, \xi_{0}\right)$, satisfies $v+p(u)>0$, we get the linear growth (see the left panel in Fig. 2),

$$
S_{A} \simeq \frac{c}{6 \beta} t+\frac{c}{6} \log \frac{\xi_{0}}{\epsilon}+S_{\mathrm{bdy}} .
$$

In this way, we may conclude that the entangled pair production occurs along the spacelike curve $v+p(u)=0$, and the propagation of the entangled pairs gives the linear
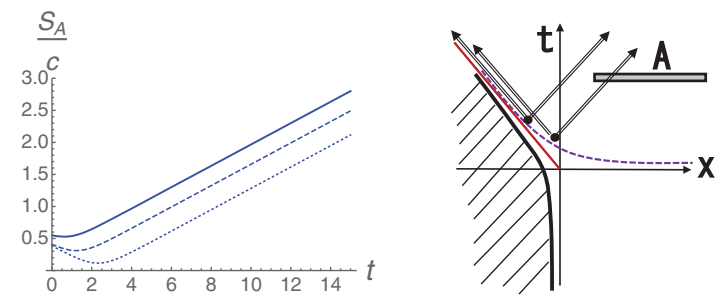

FIG. 2. The graphs in the left figure show the time evolution of entanglement entropy $S_{A}$. We choose the end point of $A$ to be $x_{0}=-t+\xi_{0}$, with $\xi_{0}=1$ (thick line), $\xi_{0}=0.1$ (dashed line) and $\xi_{0}=0.01$ (dotted line). We set $\beta=1, \epsilon=0.1$ and $S_{\text {bdy }}=0$. The right figure shows a quasiparticle picture of entanglement growth for the moving mirror. The black thick curve represents the mirror trajectory $x=Z(t)$. The purple dotted curve describes a spacelike curve defined by $v+p(u)=0$. The red line corresponds to the null line. The entangled pair production occurs on the purple dashed curve. 
growth of the entanglement entropy Eq. (12). This is sketched in the right panel of Fig. 2. We can also confirm this from the free scalar example Eq. (5), where the spacial distribution of the pair production looks like

$\left\langle 0_{\text {in }}\left|\phi\left(u_{1}, v_{1}\right) \phi\left(u_{2}, v_{2}\right) \int d \omega a_{\omega}^{\mathrm{in} \dagger} a_{\omega}^{\mathrm{in} \dagger}\right| 0_{\text {in }}\right\rangle$

$\propto \int \frac{d \omega}{\omega}\left[e^{-i \omega\left(v_{1}+p_{2}\right)}+e^{-i \omega\left(v_{2}+p_{1}\right)}-e^{-i \omega\left(v_{1}+v_{2}\right)}-e^{-i \omega\left(p_{1}+p_{2}\right)}\right]$,

where we have defined $p_{i}:=p\left(u_{i}\right)$ for brevity. The first two terms are divergent at $v_{1}+p_{2}=0$ and $v_{2}+p_{1}=0$. This shows that the entangled pairs are produced along the curve $v+p(u)=0$, and they propagate in opposite directions at the speed of light.

AdS/BCFT and entanglement entropy.-To compute $S_{A}$ for generic subsystems, we need to specify the target CFT. For holographic CFTs, we can calculate $S_{A}$ via the gravity dual of a CFT defined on a manifold $M$ with a boundary $\partial M$, i.e., boundary CFT (BCFT) [79], known as AdS/ BCFT [68]. In this description, the dual geometry is given by extending the boundary $\partial M$ into the bulk AdS, which leads to a codimension one surface $Q$, called the end of the world brane. This surface $Q$ obeys the Neumann boundary condition

$$
K_{a b}-h_{a b} K+\mathcal{T} h_{a b}=0,
$$

where $h_{a b}$ is the induced metric and $K_{a b}$ is the extrinsic curvature. The parameter $\mathcal{T}$ is the tension of the brane $Q$ and depends on the boundary condition of the CFT at $\partial M$. The condition Eq. (14) implies the presence of boundary conformal invariance. Refer to Ref. [80] for an equivalent formulation using Chern-Simons gravity, and to Ref. [81] for comparisons with CFT calculations.

We can find a gravity dual by applying the following coordinate transformation, which is a special case of Refs. [82,83] and is a bulk extension of the map Eq. (1),

$$
U=p(u), \quad V=v+\frac{p^{\prime \prime}(u)}{2 p^{\prime}(u)} z^{2}, \quad \eta=z \sqrt{p^{\prime}(u)}
$$

on Poincare $\mathrm{AdS}_{3}$

$$
d s^{2}=\frac{d \eta^{2}-d U d V}{\eta^{2}} .
$$

Using Eq. (3), this leads to the metric

$$
d s^{2}=\frac{d z^{2}}{z^{2}}+\frac{12 \pi}{c} T_{u u}(u)(d u)^{2}-\frac{1}{z^{2}} d u d v .
$$

In the Poincare $\mathrm{AdS}_{3}$ Eq. (16), by solving the boundary condition [Eq. (14)], the profile of $Q$ is given by $X=-\lambda \eta$,
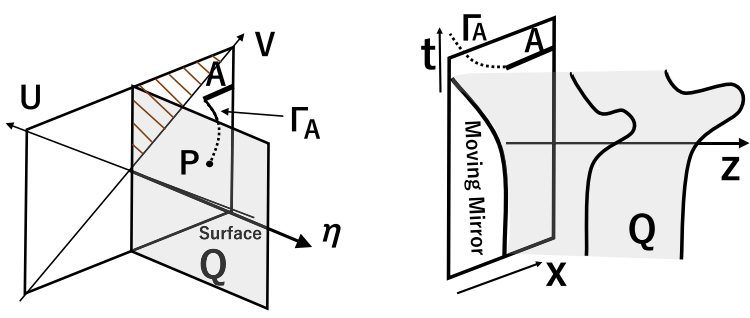

FIG. 3. Gravity dual of a moving mirror in the coordinates $(U, V, \eta)$ (left) and $(u, v, z)$ (right). We set $\mathcal{T}=0$. We also show the computation of holographic entanglement entropy.

where we have defined $\lambda=\mathcal{T} / \sqrt{1-\mathcal{T}^{2}}$, and introduced new coordinates $U=T-X$ and $V=T+X$. The metric on $Q$ is given by that of Poincare $\mathrm{AdS}_{2}$ (see the left panel in Fig. 3),

$$
d s^{2}=\frac{\left(1+\lambda^{2}\right) d \eta^{2}-d T^{2}}{\eta^{2}} .
$$

Thus, the gravity dual in terms of the $(U, V, \eta)$ coordinates is given by a part of Poincare $\mathrm{AdS}_{3}$ defined by $X+\lambda \eta>0$. Note that the surface $Q$ at the boundary $z=0$ coincides with the mirror trajectory $v=p(u)$ via the map, Eq. (15).

The gravity dual in terms of the coordinates $(u, v, z)$, which is given by the metric Eq. (17) and is sketched in the right panel of Fig. 3, only covers the region $U<0$ as $U=p(u)$ is always negative for any $u$. This is the bulk extension of the mentioned inner horizon region shown in the right panel in Fig. 1. In the coordinates $(u, v, z)$, the metric of the brane $Q$ reads

$$
\begin{aligned}
d s^{2}= & \frac{d z^{2}}{z^{2}}+\left(\frac{p^{\prime \prime}}{z p^{\prime}}+\frac{2 \lambda \sqrt{p^{\prime}}}{z^{2}}\right) d u d z \\
& +\left(\frac{p^{\prime \prime 2}}{4 p^{\prime 2}}-\frac{p^{\prime}}{z^{2}}+\frac{\lambda p^{\prime \prime}}{z \sqrt{p^{\prime}}}\right) d u^{2} .
\end{aligned}
$$

This covers only the part $T<-\lambda \eta$ of Eq. (18). In this way, the gravity dual of the moving mirror has a horizon, analogous to a single sided AdS black hole.

In general, the holographic entanglement entropy $[5,6]$ in AdS/BCFT can be computed [68] as

$$
S_{A}=\frac{1}{4 G_{N}} \operatorname{Min}_{\Gamma_{A}}\left[A\left(\Gamma_{A}\right)\right],
$$

where $A\left(\Gamma_{A}\right)$ is the length of $\Gamma_{A}$ which satisfies $\partial \Gamma_{A}=\partial A \cup \partial I_{s}$, where $I_{s}$ (i.e., island) is a region on the surface $Q$. The three-dimensional Newton constant is denoted by $G_{N}$. The minimum in Eq. (20) is taken over all possible choices of $I_{s}$ and $\Gamma_{A}$. When $A$ is an interval $\left[x_{0}, x_{1}\right]$ at time $t$, there appear to be two candidates for $\Gamma_{A}$. One is a connected geodesic between $x=x_{0}$ and $x=x_{1}$. The other one is a union of two disconnected ones, each of which 

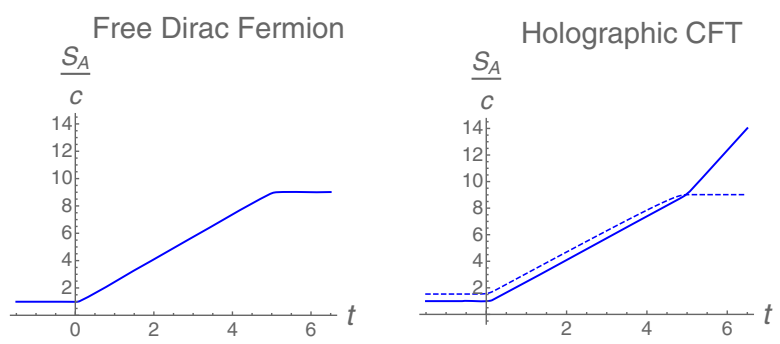

FIG. 4. The time evolution of entanglement entropy for a moving mirror [Eq. (6)] in free Dirac fermion CFT (left) and in holographic CFT (right). Here, we set the subsystem to be $A=[Z(t)+0.1, Z(t)+10]$ with $\beta=0.1, \epsilon=0.1$ and $S_{\text {bdy }}=0$. On the right, the thick line and the dashed line show the disconnected and connected entanglement entropy, respectively.

departs from $x=x_{0}$ (or $x=x_{1}$ ) and ends on $Q$, respectively.

In our setup, they are explicitly given by $S_{A}=\operatorname{Min}\left[S_{A}^{\text {con }}, S_{A}^{\text {dis }}\right]$, where the disconnected and connected geodesic contributions $S_{A}^{\text {dis }}$ and $S_{A}^{\text {con }}$ read

$$
\begin{aligned}
S_{A}^{\mathrm{dis}}= & \frac{c}{6} \log \frac{t+x_{0}-p\left(t-x_{0}\right)}{\epsilon \sqrt{p^{\prime}\left(t-x_{0}\right)}} \\
& +\frac{c}{6} \log \frac{t+x_{1}-p\left(t-x_{1}\right)}{\epsilon \sqrt{p^{\prime}\left(t-x_{1}\right)}}+2 S_{\mathrm{bdy}}, \\
S_{A}^{\mathrm{con}}= & \frac{c}{6} \log \frac{\left(x_{1}-x_{0}\right)\left[p\left(t-x_{0}\right)-p\left(t-x_{1}\right)\right]}{\epsilon^{2} \sqrt{p^{\prime}\left(t-x_{0}\right) p^{\prime}\left(t-x_{1}\right)}} .
\end{aligned}
$$

The boundary entropy is a function of the tension and is given by $S_{\text {bdy }}=(c / 6) \log \sqrt{(1+\mathcal{T}) /(1-\mathcal{T})}$. When $A$ is semi-infinite, i.e., $x_{1} \rightarrow \infty$, we always have $S_{A}=S_{A}^{\text {dis }}$, and this reproduces Eq. (8). When $A$ is a finite interval, $S_{A}^{\text {dis }}$ is initially favored and this gives the linear growth as in Eq. (12). At later time, $S_{A}^{\text {con }}$ is favored and this leads to a saturation as depicted in the right panel of Fig. 4. We have also plotted $S_{A}$ for the massless free Dirac fermion case, which is shown in the left panel of Fig. 4.

Page curve from a moving mirror.-A typical moving mirror model which mimics an evaporating black hole is found by setting

$$
p(u)=-\beta \log \left(1+e^{-\frac{u}{\beta}}\right)+\beta \log \left(1+e^{\frac{u-u_{0}}{\beta}}\right),
$$

whose mirror trajectory $x=Z(t)$ and energy flux $T_{u и}$ are depicted in Fig. 5. When $\beta$ is small, we can approximate the trajectory as $Z(t) \simeq 0$ for $t<0, \quad Z(t) \simeq-t$ for $0<t<u_{0} / 2$, and $Z(t)=-u_{0} / 2$ for $t>u_{0} / 2$. The energy flux is nonvanishing, $T_{u u} \simeq c / 48 \pi \beta^{2}$, namely, only for the period $0<u<u_{0}$.

We can again calculate the holographic entanglement entropy $S_{A}=\operatorname{Min}\left[S_{A}^{\text {con }}, S_{A}^{\text {dis }}\right]$, using Eq. (21) as plotted in Fig. 6. In particular, when $A$ is a semi-infinite line, $S_{A}$ takes the form of the Page curve. For $0<t<u_{0} / 4, S_{A}$ grows
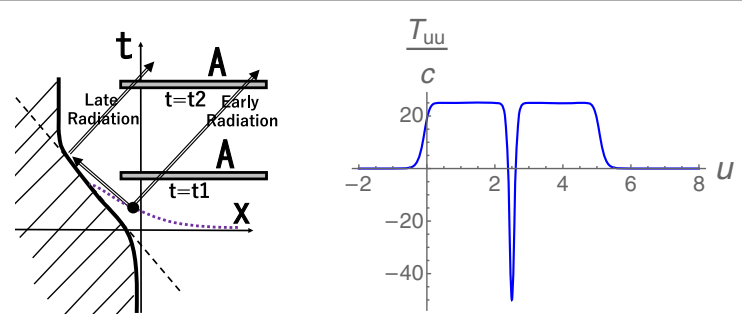

FIG. 5. The profile of a moving mirror, with the creation of entangled pairs and their reflection at the mirror, is depicted in the left picture. The right graph is the energy density $T_{u и}$ plotted as a function of $u$. We set $\beta=0.1$ and $u_{0}=5$.

linearly $d S_{A} / d t \simeq c / 6 \beta$, and for $u_{0} / 4<t<u_{0} / 2$, it decreases linearly, i.e., $d S_{A} / d t \simeq-(c / 6 \beta)$. Note that as is clear from Fig. 6, the disconnected result $S^{\text {dis }}$ gives the dominant contribution (refer to Ref. [70] for an earlier calculation of the connected result $S^{\text {con }}$ ).

The initial linear growth of $S_{A}$ can be understood as in the previous example [Eq. (12)] by considering entangled pair production along the curve $v+p(u)=0$. Moreover, the linear decay of $S_{A}$ is explained by reflections of the left moving partner, as shown in the left picture of Fig. 5. When $A$ is a finite interval, we have two Page peaks. The first peak occurs when only the originally right moving particles are crossing $A$. The second peak appears when only the reflected particles are crossing $A$.

Brane world gravity and island.-The gravity dual of our moving mirror setup can be interpreted in an alternative way by regarding the surface $Q$ as an end of the world brane in the brane world setup [84-86]. This situation is depicted in the left panel of Fig. 7. According to this interpretation, the CFT defined in the region $x \geq Z(t)$ will be coupled to a two-dimensional gravity theory on $Q$. By estimating the effective Newton constant on $Q$ via KaluzaKlein reduction $[44,84-86]$, which we denote by $G_{N}^{(Q)}$, we can show

$$
\frac{1}{4 G_{N}^{(Q)}}=S_{\mathrm{bdy}}
$$
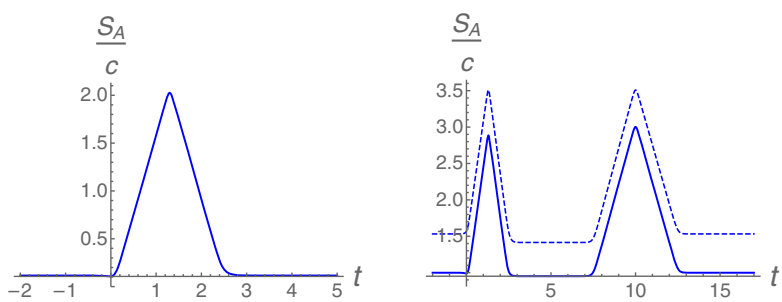

FIG. 6. The time evolution of holographic entanglement entropy follows the Page curve. We choose $A$ to be a semi-infinite line $A=[Z(t)+0.1, \infty]$ in the left and a finite interval $A=$ $[Z(t)+0.1, Z(t)+10]$ in the right. The thick and dashed curves describe $S_{A}^{\text {dis }}$ and $S_{A}^{\text {con }}$, respectively. We set $\beta=0.1, u_{0}=5, \epsilon=$ 0.1 and $S_{\text {bdy }}=0$. 

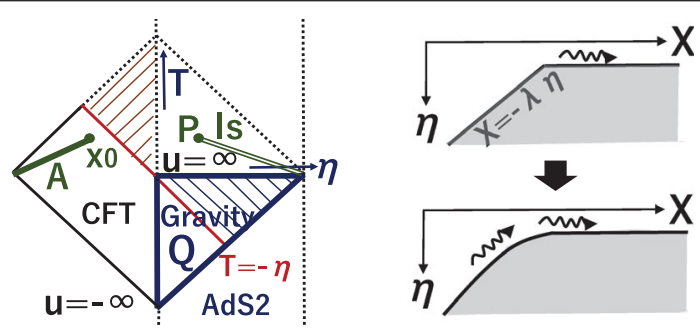

FIG. 7. The left picture shows the global spacetime of a CFT (left triangle) and the brane world gravity on $Q$ (right triangle), which are attached along the mirror trajectory. The island (doubled green line) is shown as well. The right picture sketches the deformation of the gravity dual of the moving mirror (top) into that with black hole radiation (bottom).

The gravitational entropy of $\mathrm{AdS}_{2}$, i.e., the brane $Q$, will thus be equal to the boundary entropy $S_{\text {bdy }}$.

We can regard $S_{A}$ in Eq. (8) as the entanglement entropy of the subregion $A$ in a system consisting of a CFT on $x \geq Z(t)$ and a gravitational theory on $Q$, glued along the moving mirror. Then, we can interpret the first and second term in Eq. (8) as the bulk entropy contribution $S_{A \cup I_{s}}^{\text {bulk }}$ and the area term $\operatorname{Area}\left(\partial I_{s}\right) / 4 G_{N}$, respectively, in the island formula [9-13]. Note that here we use the standard formula for computing holographic entanglement entropy without invoking the quantum extremal surface prescription. The density matrix under consideration is pure and the radiation is manifestly unitary.

In our moving mirror model, the entropy [Eq. (8)] shows linear growth in the region defined by the equation $v+p(u)>0$. This part in the entanglement entropy would arise from the island. This region is not covered by the coordinate patch [Eq. (19)], as sketched in the left panel of Fig. 7.

An interesting feature in our setup is the entanglement between the gravitational theory on $Q$ and the CFT by the amount [Eq. (23)]. Also it is important to note that the presence of energy flux from the boundary is different from standard BCFTs (Cardy states [87]) which have no energy flux condition $T(w)-\bar{T}(\bar{w})=0$ at the boundary.

The radiation in the CFT looks similar to the setups [9-13], where the Page curve was derived. However, unlike these, in our BCFT model, we find that there is no radiation present in the gravitational system on $Q$. That is, the flux does not come from the gravitational system, but is created on the boundary. Indeed, the holographic energy stress tensor [88] on $Q$ is proportional to $h_{a b}$ as it follows from Eq. (14). Hence, it can just be regarded as a negative cosmological constant. The absence of radiation from $Q$ is obviously consistent with the fact that the mirror is completely reflective. We can regard our analysis as a derivation of the Page curve in the strong coupling regime of gravity, while Refs. [9-13] focus on the weak coupling regime.

Indeed, by changing the profile of the brane $Q$, we can deform our setup of the moving mirror such that it incorporates radiation resulting from the gravitational sector on $Q$, see right panel of Fig. 7. If we modify the surface $Q$ to make it close to the standard AdS boundary located at $z=\epsilon$, the matter energy stress tensor on $Q$ will be approximated by Eq. (7). This provides a special and concrete example of the setup considered in Ref. [11], see also Refs. [15-17,37,42,44,48] for related works. The holographic dual of this modified setup is a two-dimensional CFT coupled to two-dimensional gravity. One advantage of our procedure is that our calculation based on the BCFT analysis is much easier than the one based on the conformal welding problem $[10,13]$. An interesting future direction will be relating the two realizations in an explicit way [89].

Conclusion.-In this Letter, we have presented a gravity dual of two-dimensional CFT with a moving mirror, which mimics black hole formation and evaporation. We have explicitly calculated the time evolution of entanglement entropy in the presence of the mirror. We have found that it follows the ideal Page curve. This can be explained by the creation of entangled particles, their propagation, and reflection from the mirror. We have also discussed that modifying the profile of the end of the world brane in the gravity dual results in a model for two-dimensional black hole radiation. In order to understand unitary evolution for realistic black hole evaporation, we will have to incorporate the singularity. We expect that the presence of spacelike boundaries in the CFT and its gravity dual $[44,48]$ will be relevant [89].

We are grateful to Tatsuma Nishioka, Kotaro Tamaoka, and Tomonori Ugajin for useful comments on a draft of this Letter. I. A., Y. K., and Z. W. are supported by the Japan Society for the Promotion of Science (JSPS). I. A. is supported by the Alexander von Humboldt (AvH) foundation. I. A. and T. T. are supported by Grant-in-Aid for JSPS Fellows No. 19F19813. Y. K. is supported by Grant-in-Aid for JSPS Fellows No. 18J22495. N. S. is also supported by JSPS KAKENHI Grant No. JP19K14721. T. T. is supported by the Simons Foundation through the "It from Qubit" collaboration. T. T. is supported by Inamori Research Institute for Science and World Premier International Research Center Initiative (WPI Initiative) from the Japan Ministry of Education, Culture, Sports, Science and Technology (MEXT). N.S. and T. T. are supported by JSPS Grant-in-Aid for Scientific Research (A) No. 16H02182. T. T. is also supported by JSPS Grantin-Aid for Challenging Research (Exploratory) 18K18766. Z.W. is supported by the ANRI Fellowship and Grant-inAid for JSPS Fellows No. 20J23116.

[1] S. W. Hawking, Particle creation by black holes, Commun. Math. Phys. 43, 199 (1975); 46, 206 (1976).

[2] N. Birrell and P. Davies, Quantum Fields in Curved Space, Cambridge Monographs on Mathematical Physics (Cambridge University Press, Cambridge, England, 1984). 
[3] P. Davies and S. Fulling, Radiation from a moving mirror in two-dimensional space-time conformal anomaly, Proc. R. Soc. A 348, 393 (1976).

[4] D. N. Page, Average Entropy of a Subsystem, Phys. Rev. Lett. 71, 1291 (1993); Information in Black Hole Radiation, Phys. Rev. Lett. 71, 3743 (1993).

[5] S. Ryu and T. Takayanagi, Holographic Derivation of Entanglement Entropy from AdS/CFT, Phys. Rev. Lett. 96, 181602 (2006).

[6] V.E. Hubeny, M. Rangamani, and T. Takayanagi, A covariant holographic entanglement entropy proposal, J. High Energy Phys. 07 (2007) 062.

[7] T. Faulkner, A. Lewkowycz, and J. Maldacena, Quantum corrections to holographic entanglement entropy, J. High Energy Phys. 11 (2013) 074.

[8] N. Engelhardt and A. C. Wall, Quantum extremal surfaces: Holographic entanglement entropy beyond the classical regime, J. High Energy Phys. 01 (2015) 073.

[9] G. Penington, Entanglement wedge reconstruction and the information paradox, J. High Energy Phys. 09 (2020) 002.

[10] A. Almheiri, N. Engelhardt, D. Marolf, and H. Maxfield, The entropy of bulk quantum fields and the entanglement wedge of an evaporating black hole, J. High Energy Phys. 12 (2019) 063.

[11] A. Almheiri, R. Mahajan, J. Maldacena, and Y. Zhao, The page curve of Hawking radiation from semiclassical geometry, J. High Energy Phys. 03 (2020) 149.

[12] G. Penington, S. H. Shenker, D. Stanford, and Z. Yang, Replica wormholes and the black hole interior, arXiv:1911.11977.

[13] A. Almheiri, T. Hartman, J. Maldacena, E. Shaghoulian, and A. Tajdini, Replica wormholes and the entropy of Hawking radiation, J. High Energy Phys. 05 (2020) 013.

[14] C. Akers, N. Engelhardt, and D. Harlow, Simple holographic models of black hole evaporation, J. High Energy Phys. 08 (2020) 032.

[15] A. Almheiri, R. Mahajan, and J. Maldacena, Islands outside the horizon, arXiv:1910.11077; A. Almheiri, R. Mahajan, and J.E. Santos, Entanglement islands in higher dimensions, SciPost Phys. 9, 001 (2020).

[16] M. Rozali, J. Sully, M. Van Raamsdonk, C. Waddell, and D. Wakeham, Information radiation in BCFT models of black holes, J. High Energy Phys. 05 (2020) 004.

[17] H. Z. Chen, Z. Fisher, J. Hernandez, R. C. Myers, and S. M. Ruan, Information flow in black hole evaporation, J. High Energy Phys. 03 (2020) 152.

[18] R. Bousso and M. Tomašević, Unitarity from a smooth horizon?, Phys. Rev. D 102, 106019 (2020).

[19] Y. Kusuki, Y. Suzuki, T. Takayanagi, and K. Umemoto, Looking at shadows of entanglement wedges, Prog. Theor. Exp. Phys. 2020, 11B105 (2020).

[20] J. Pollack, M. Rozali, J. Sully, and D. Wakeham, Eigenstate Thermalization and Disorder Averaging in Gravity, Phys. Rev. Lett. 125, 021601 (2020).

[21] H. Liu and S. Vardhan, A dynamical mechanism for the Page curve from quantum chaos, arXiv:2002.05734.

[22] D. Marolf and H. Maxfield, Transcending the ensemble: Baby universes, spacetime wormholes, and the order and disorder of black hole information, J. High Energy Phys. 08 (2020) 044; Observations of Hawking radiation: The page curve and baby universes, arXiv:2010.06602.
[23] L. Piroli, C. Sünderhauf, and X. L. Qi, A random unitary circuit model for black hole evaporation, J. High Energy Phys. 04 (2020) 063.

[24] V. Balasubramanian, A. Kar, O. Parrikar, G. Sárosi, and T. Ugajin, Geometric secret sharing in a model of Hawking radiation, arXiv:2003.05448.

[25] H. Verlinde, ER = EPR revisited: On the entropy of an Einstein-Rosen bridge, arXiv:2003.13117.

[26] Y. Chen, X. L. Qi, and P. Zhang, Replica wormhole and information retrieval in the SYK model coupled to Majorana chains, J. High Energy Phys. 06 (2020) 121.

[27] F. F. Gautason, L. Schneiderbauer, W. Sybesma, and L. Thorlacius, Page curve for an evaporating black hole, J. High Energy Phys. 05 (2020) 091.

[28] T. Anegawa and N. Iizuka, Notes on islands in asymptotically flat $2 \mathrm{~d}$ dilaton black holes, J. High Energy Phys. 07 (2020) 036.

[29] S. B. Giddings and G. J. Turiaci, Wormhole calculus, replicas, and entropies, J. High Energy Phys. 09 (2020) 194.

[30] K. Hashimoto, N. Iizuka, and Y. Matsuo, Islands in Schwarzschild black holes, J. High Energy Phys. 06 (2020) 085.

[31] T. Hartman, E. Shaghoulian, and A. Strominger, Islands in asymptotically flat 2D gravity, J. High Energy Phys. 07 (2020) 022.

[32] C. A. Agón, S. F. Lokhande, and J.F. Pedraza, Local quenches, bulk entanglement entropy and a unitary Page curve, J. High Energy Phys. 08 (2020) 152.

[33] T. J. Hollowood and S. P. Kumar, Islands and page curves for evaporating black holes in JT gravity, J. High Energy Phys. 08 (2020) 094.

[34] C. Krishnan, V. Patil, and J. Pereira, Page curve and the information paradox in flat space, arXiv:2005.02993.

[35] M. Alishahiha, A. Faraji Astaneh, and A. Naseh, Island in the presence of higher derivative terms, arXiv:2005.08715.

[36] H. Geng and A. Karch, Massive islands, J. High Energy Phys. 09 (2020) 121.

[37] H. Z. Chen, R. C. Myers, D. Neuenfeld, I. A. Reyes, and J. Sandor, Quantum extremal islands made easy, Part I: Entanglement on the brane, J. High Energy Phys. 10 (2020) 166; Quantum extremal islands made easy, Part II: Black holes on the brane, J. High Energy Phys. 12 (2020) 025; , Quantum extremal Islands made easy, PartIII: Complexity on the brane, arXiv:2010.16398.

[38] A. Almheiri, T. Hartman, J. Maldacena, E. Shaghoulian, and A. Tajdini, The entropy of Hawking radiation, arXiv:2006.06872.

[39] T. Li, J. Chu, and Y. Zhou, Reflected entropy for an evaporating black hole, J. High Energy Phys. 11 (2020) 155.

[40] V. Chandrasekaran, M. Miyaji, and P. Rath, Including contributions from entanglement islands to the reflected entropy, Phys. Rev. D 102, 086009 (2020).

[41] D. Bak, C. Kim, S. H. Yi, and J. Yoon, Unitarity of entanglement and islands in two-sided Janus black holes, [arXiv:2006.11717.

[42] R. Bousso and E. Wildenhain, Gravity/ensemble duality, Phys. Rev. D 102, 066005 (2020).

[43] X. Dong, X. L. Qi, Z. Shangnan, and Z. Yang, Effective entropy of quantum fields coupled with gravity, J. High Energy Phys. 10 (2020) 052. 
[44] I. Akal, Y. Kusuki, T. Takayanagi, and Z. Wei, Codimension two holography for wedges, Phys. Rev. D 102, 126007 (2020).

[45] N. Engelhardt, S. Fischetti, and A. Maloney, Free energy from replica wormholes, arXiv:2007.07444.

[46] A. Karlsson, Replica wormhole and island incompatibility with monogamy of entanglement, arXiv:2007.10523.

[47] H. Z. Chen, Z. Fisher, J. Hernandez, R. C. Myers, and S. M. Ruan, Evaporating black holes coupled to a thermal bath, J. High Energy Phys. 01 (2021) 065.

[48] Y. Chen, V. Gorbenko, and J. Maldacena, Bra-ket wormholes in gravitationally prepared states, arXiv:2007.16091.

[49] T. Hartman, Y. Jiang, and E. Shaghoulian, Islands in cosmology, J. High Energy Phys. 11 (2020) 111.

[50] C. Murdia, Y. Nomura, and P. Rath, Coarse-graining holographic states: A semiclassical flow in general spacetimes, Phys. Rev. D 102, 086001 (2020).

[51] A. Altland and J. Sonner, Late time physics of holographic quantum chaos, arXiv:2008.02271.

[52] V. Balasubramanian, A. Kar, and T. Ugajin, Islands in de Sitter space, arXiv:2008.05275; Entanglement between two disjoint universes, arXiv:2008.05274.

[53] W. Sybesma, Pure de Sitter space and the island moving back in time, arXiv:2008.07994.

[54] D. Stanford, More quantum noise from wormholes, arXiv:2008.08570.

[55] Y. Ling, Y. Liu, and Z. Y. Xian, Island in charged black holes, arXiv:2010.00037.

[56] J. Chakravarty, Overcounting of interior excitations: A resolution to the bags of gold paradox in AdS, arXiv:2010.03575.

[57] A. Bhattacharya, A. Chanda, S. Maulik, C. Northe, and S. Roy, Topological shadows and complexity of islands in multiboundary wormholes, arXiv:2010.04134.

[58] D. Harlow and E. Shaghoulian, Global symmetry, Euclidean gravity, and the black hole information problem, arXiv:2010.10539.

[59] Y. Chen and H.W. Lin, Signatures of global symmetry violation in relative entropies and replica wormholes, arXiv:2011.06005.

[60] J. Kirklin, Islands and Uhlmann phase: Explicit recovery of classical information from evaporating black holes, arXiv:2011.07086.

[61] K. Goto, T. Hartman, and A. Tajdini, Replica wormholes for an evaporating 2D black hole, arXiv:2011.09043.

[62] P. S. Hsin, L. V. Iliesiu, and Z. Yang, A violation of global symmetries from replica wormholes and the fate of black hole remnants, arXiv:2011.09444.

[63] Y. Nomura, Spacetime and universal soft modes-Black holes and beyond, Phys. Rev. D 101, 066024 (2020); Black hole interior in unitary gauge construction, arXiv:2010.15827.

[64] A. Laddha, S. G. Prabhu, S. Raju, and P. Shrivastava, The holographic nature of null infinity, arXiv:2002.02448.

[65] I. Akal, Universality, intertwiners and black hole information, arXiv:2010.12565.

[66] J. M. Maldacena, The large $N$ limit of superconformal field theories and supergravity, Adv. Theor. Math. Phys. 2, 231 (1998); Int. J. Theor. Phys. 38, 1113 (1999).
[67] A. Karch and L. Randall, Open and closed string interpretation of SUSY CFT's on branes with boundaries, J. High Energy Phys. 06 (2001) 063.

[68] T. Takayanagi, Holographic Dual of BCFT, Phys. Rev. Lett. 107, 101602 (2011); M. Fujita, T. Takayanagi, and E. Tonni, Aspects of AdS/BCFT, J. High Energy Phys. 11 (2011) 043.

[69] E. Bianchi and M. Smerlak, Entanglement entropy and negative energy in two dimensions, Phys. Rev. D 90, 041904(R) (2014).

[70] M. Hotta and A. Sugita, The fall of black hole firewall: Natural nonmaximal entanglement for page curve, Prog. Theor. Exp. Phys. 2015, 123B04 (2015).

[71] M. R. R. Good, K. Yelshibekov, and Y. C. Ong, On horizonless temperature with an accelerating mirror, J. High Energy Phys. 03 (2017) 013.

[72] P. Chen and D.h. Yeom, Entropy evolution of moving mirrors and the information loss problem, Phys. Rev. D 96, 025016 (2017).

[73] M. R. R. Good, E. V. Linder, and F. Wilczek, Moving mirror model for quasithermal radiation fields, Phys. Rev. D 101, 025012 (2020).

[74] P. Calabrese and J. L. Cardy, Evolution of entanglement entropy in one-dimensional systems, J. Stat. Mech. (2005) P04010.

[75] P. Calabrese and J. L. Cardy, Entanglement entropy and quantum field theory, J. Stat. Mech. (2004) P06002; Entanglement entropy and conformal field theory, J. Phys. A 42, 504005 (2009).

[76] C. Holzhey, F. Larsen, and F. Wilczek, Geometric and renormalized entropy in conformal field theory, Nucl. Phys. B424, 443 (1994).

[77] H. Casini and M. Huerta, Entanglement entropy in free quantum field theory, J. Phys. A 42, 504007 (2009).

[78] I. Affleck and A. W. W. Ludwig, Universal Noninteger 'Ground State Degeneracy' in Critical Quantum Systems, Phys. Rev. Lett. 67, 161 (1991).

[79] J. L. Cardy, Boundary conformal field theory, arXiv:hep-th/ 0411189.

[80] T. Takayanagi and T. Uetoko, Chern-Simons gravity dual of BCFT, arXiv:2011.02513.

[81] J. Sully, M. Van Raamsdonk, and D. Wakeham, BCFT entanglement entropy at large central charge and the black hole interior, arXiv:2004.13088.

[82] M. Banados, Three-dimensional quantum geometry and black holes, AIP Conf. Proc. 484, 147 (1999).

[83] M. M. Roberts, Time evolution of entanglement entropy from a pulse, J. High Energy Phys. 12 (2012) 027.

[84] L. Randall and R. Sundrum, A Large Mass Hierarchy from a Small Extra Dimension, Phys. Rev. Lett. 83, 3370 (1999).

[85] L. Randall and R. Sundrum, An Alternative to Compactification, Phys. Rev. Lett. 83, 4690 (1999).

[86] A. Karch and L. Randall, Locally localized gravity, J. High Energy Phys. 05 (2001) 008.

[87] J. L. Cardy, Boundary conditions, fusion rules and the Verlinde formula, Nucl. Phys. B324, 581 (1989).

[88] V. Balasubramanian and P. Kraus, A stress tensor for Anti-de Sitter gravity, Commun. Math. Phys. 208, 413 (1999).

[89] I. Akal, Y. Kusuki, N. Shiba, T. Takayanagi, and Z. Wei (to be published) 